\title{
Targeting influenza vaccinations of children
}

\author{
Ville Peltola MD PhD, Olli Ruuskanen MD PhD, Terho Heikkinen MD PhD
}

See related research article by Hoen and colleagues at www.cmaj.ca/lookup/doi/10.1503/cmaj.110241 and synopsis on page 1492 .

Competing interests:

Ville Peltola has been paid as a consultant by Novartis and his institution has grants or grants pending from GlaxoSmithKline. Olli Ruuskanen has been paid as a consultant by Novartis Vaccines and Abbott. Terho Heikkinen has been paid as a consultant by Novartis, AstraZeneca, GlaxoSmithKline and Solvay.

This article was solicited and has not been peer reviewed.

Correspondence to: Dr. Ville Peltola, ville.peltola@utu.fi

CMAJ 2011. DOI:10.1503 /cmaj.111055

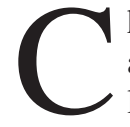
hildren younger than two years of age are a risk group for severe influenza leading to hospital admission. Preschool- and school-aged children also have a high incidence of influenza during yearly epidemics. The benefits of influenza vaccination in these age groups have not been determined as clearly as in younger children. In the accompanying research article by Hoen and colleagues, the authors report a reduction in influenza-like illness in two- to four-year-old children in Boston, Massachusetts, after inclusion of this age group in the immunization program, but not in Montréal, Quebec, where vaccination was not recommended in this age group.

The effects of changes in general vaccination recommendations on disease rates can be estimated by observational, ecologic studies. In the study by Hoen and coauthors, comparisons were done not only between periods before and after a change in vaccination policy, but also between two areas with a change in policy only in one (Boston). Observational studies such as this one measure real-life effects of vaccination, with the rates of coverage that were actually achieved and parameters used to calculate the burden of disease that reflect the use of health care. Importantly, observational studies detect effects at the population level. Hoen and colleagues report statistically significant decreases in influenza-like illness not only in the age group of two to four years in which vaccinations were recommended, but also in children of other ages. This may have been caused by herd immunity (i.e., decreased transmission of influenza), increased rates of

\section{- Ker pOINTS}

- Inclusion of two- to four-year-old children in the influenza immunization program in the United States resulted in a reduction in influenza-like illness in this and other pediatric age groups.

- A caveat in these results is that the correlation of influenza-like illness with laboratory-documented influenza is poor in children.

- Broad recommendations for influenza vaccination could lead to population-level benefits by inducing herd immunity and increasing vaccine coverage in risk groups.

- Vaccines for seasonal influenza are safe and effective in children. vaccination in other age groups or other nonspecific effects.

In observational studies, the effects of vaccination may get confused with effects of other factors on the rate of disease. Comparison of two centres with different vaccination policies adds a level of control to Hoen and colleagues' study. The authors also used sophisticated statistical methods to model their observations. However, influenza is a particularly challenging object for observational studies of vaccination. There are two main reasons for this. First, by clinical examination only, it's often impossible to differentiate infections from an influenza virus from infections from other respiratory viruses. Second, year-to-year variations in influenza epidemics are substantial. Furthermore, the match of vaccine with the circulating influenza viruses is variable.

Influenza-like illness is often used as a surrogate for laboratory-documented influenza. Usually, influenza-like illness is defined by acute onset of fever and cough with or without other symptoms or signs. The problem is that no matter what definition for influenza-like illness is used, its correlation with laboratory-documented influenza is poor in children. In a prospective cohort study of children, only $38 \%$ of cases of influenza A or B virus detected by culture were diagnosed clinically, and only $32 \%$ of children with a clinical diagnosis of influenza had the diagnosis confirmed in the laboratory. ${ }^{2}$ The nonspecific nature of influenza-like illness is seen by comparing Figures 1 and 2 in the paper by Hoen and colleagues. Laboratory detections of influenza A and B viruses form sharp peaks of varying height in the winter and are absent in the summer, whereas visits for influenza-like illness show broad peaks each winter and are not absent in the summer. Different epidemiologic patterns of influenza viruses and visits for influenza-like illness show that a considerable part of the latter has to be caused by viruses other than influenza A or B.

Active circulation of influenza viruses in the study population and a good match with the vaccine strains are prerequisites for any study to

All editorial matter in CMAJ represents the opinions of the authors and not necessarily those of the Canadian Medical Association. 
show the effectiveness of influenza vaccines. The effectiveness of influenza vaccines in children is good in studies conducted under these conditions. However, there has been some degree of confusion about the efficacy of influenza vaccination in young children, as a consequence of the variation in annual influenza epidemics and the use of influenza-like illness as an end point in some studies. Hoberman and colleagues reported effectiveness of $66 \%$ against culture-confirmed influenza in the first season of their study but no effectiveness during the second season when influenza activity was low. ${ }^{3}$ This finding was later erroneously referred to as providing evidence that influenza vaccines are ineffective in children. ${ }^{4}$ Heinonen and coauthors reported recently an effectiveness of $66 \%$ of trivalent inactivated influenza vaccine given as part of the routine national vaccination program in children in Finland between nine months and three years of age. ${ }^{5}$ This effectiveness rate was found despite a lineage-level mismatch between the vaccine strain and circulating strain of influenza B virus during the study; the effectiveness of the vaccine against influenza A virus was as high as $84 \%$.

Recommendations for use of influenza vaccines need to consider the potential benefits and harms both at the individual and population levels. The safety record of nonadjuvanted, inactivated influenza vaccines is excellent. ${ }^{6}$ Newer, live attenuated influenza vaccines are also safe and effective in children without history of wheezing or asthma, in those age groups where they are indicated for use.?

Although children younger than two years, elderly adults, pregnant women and people with chronic illnesses are well-defined risk groups for severe influenza, children of other ages and healthy adults would also benefit from influenza vaccinations in the form of decreases in outpatient visits, antibiotic and antiviral consumption, absenteeism from school and jobs and, to a lesser degree, hospital admissions and deaths. ${ }^{8}$ Expansion of vaccinations should also have beneficial effects at the population level, because vaccinated people do not transmit influenza viruses further.

The practical benefits of a broad recommendation for influenza vaccination may be even more important. Coverage with influenza vaccines is quite low in most countries, even in the targeted risk groups. If the vaccine could be offered to all children or all members of households regardless of age or chronic illnesses, the organization of vaccine delivery may be easier for both health care providers and citizens. In addition, low price of inactivated influenza vac- cines makes broad recommendations feasible.

The study by Hoen and coauthors provides supporting evidence for the decision to expand the recommendations for influenza vaccination in the United States, and more recently in Canada, to cover older children. These findings should also be taken into consideration in other countries. For example, few countries in Europe recommend influenza vaccinations even for young children.

\section{References}

1. Hoen AG, Buckeridge DL, Charland KML, et al. Effect of expanded US recommendations for seasonal influenza vaccination: comparison of two pediatric emergency departments in the United States and Canada. CMAJ 2011;183:E1021-8.

2. Peltola V, Reunanen T, Ziegler T, et al. Accuracy of clinical diagnosis of influenza in outpatient children. Clin Infect Dis 2005;41:1198-200.

3. Hoberman A, Greenberg DP, Paradise JL, et al. Effectiveness of inactivated influenza vaccine in preventing acute otitis media in young children: a randomized controlled trial. JAMA 2003;290: 1608-16.

4. Jefferson T, Rivetti A, Harnden A, et al. Vaccines for preventing influenza in healthy children [review]. Cochrane Database Syst Rev 2008;(2):CD004879.

5. Heinonen S, Silvennoinen H, Lehtinen P, et al. Effectiveness of inactivated influenza vaccine in children aged 9 months to 3 years: an observational cohort study. Lancet Infect Dis 2011;11: 23-9.

6. Hambidge SJ, Glanz JM, France EK, et al. Safety of trivalent inactivated influenza vaccine in children 6 to 23 months old. JAMA 2006;296:1990-7.

7. Belshe RB, Edwards KM, Vesikari T, et al. Live attenuated versus inactivated influenza vaccine in infants and young children. N Engl J Med 2007;356:685-96.

8. Molinari NA, Ortega-Sanchez IR, Messonnier ML, et al. The annual impact of seasonal influenza in the US: measuring disease burden and costs. Vaccine 2007;25:5086-96.

Affiliations: Ville Peltola, Olli Ruuskanen and Terho Heikkinen are with the Department of Pediatrics, Turku University Hospital, Turku, Finland

Contributors: All of the authors contributed to drafting and revising the manuscript and approved the final version submitted for publication.

\section{Call for papers: CMAJ Holiday Reading}

Hilarity and good humour ... help enormously in both the study and the practice of medicine. William Osler

Too hot to handle? This is the shocking sort of research that we will seriously consider for CMAJ's 2011 Holiday Reading section. Articles should be no longer than 1200 words; photographs and illustrations are welcome. Please submit your twisted research, satire and culturally sensitive missives online at http://mc.manuscriptcentral.com/cmaj, specifying that it is for the Holiday Reading section.

Deadline: October 3, 2011. 